\title{
Raman spectrum of group IV nanowires: influence of temperature
}

\author{
J.Anaya $^{1}$, C.Prieto ${ }^{1}$, J.Souto ${ }^{1}$, J. Jiménez ${ }^{1}$, A.Rodríguez ${ }^{2}$, J.Sangrador ${ }^{2}$,
}

T.Rodríguez ${ }^{2}$

${ }^{1}$ Optronlab Group, Dpto. Física de la Materia Condensada, Centro I+D, Univ. de Valladolid, Paseo de Belén 1, 47011 Valladolid, Spain

\author{
${ }^{2}$ Tecnología Electrónica, E.T.S.I.T., Universidad Politécnica de Madrid, 28040 Madrid, \\ Spain
}

\begin{abstract}
Group IV semiconductor nanowires are characterized by Raman spectroscopy. The results are analyzed in terms of the heating induced by the laser beam on the nanowires. By solving the heat transport equation one can simulate the temperature reached by the NWs under the exposure to a laser beam. The results are illustrated with $\mathrm{Si}$ and $\mathrm{Si}_{1-\mathrm{x}} \mathrm{Ge}_{\mathrm{x}}$ nanowires. Both bundles of nanowires and individual nanowires are studied. The main experimental conditions contributing to the nanowire heating are discussed.
\end{abstract}

\section{INTRODUCTION}

Nanoscaled semiconductor structures present a high potential for the fabrication of new devices because of their unique optical and electrical properties (1). In particular nanowires (NWs) will be the building blocks for future advanced electronic, and optoelectronic nanodevices (1). A great deal of characterization effort has been devoted to the study of the NWs properties, which present a strong dependence with its dimension. One of the main issues regarding the NWs concerns its thermal management. In fact, NWs are systems with lower thermal conductivity than their bulk counterparts (2), on the other hand they are usually standing in air; therefore, the thermal dissipation is very slow constituting a serious drawback for the practical use of these structures. The study of the thermal behaviour of semiconductor NWs is a crucial issue for the future application of the NWs. In this sense, Raman spectroscopy is a very powerful tool to study the properties of NWs, since it can provide information about phonon confinement associated with the reduced size $(3,4)$, but also it is very sensitive to temperature (5). Both effects, phonon confinement and temperature, need to be separated in order to give a reliable interpretation of the Raman spectrum of NWs. The analysis of the Raman spectrum of nanosized structures is based on the phenomenological confinement model introduced by Ley et al (3), further extended to NWs by Campbell et al (4). In the case of Si NWs there is a great amount of controversy because of the discrepancies between the expected and measured lineshapes of the one phonon bands, which does not permit to provide accurate values for the NWs diameter (6-10). The one phonon Raman lineshape can be contributed by other factors, e.g. dispersion of NW diameters (6), Fano broadening from photogenerated carriers (7), and laser induced heating of the NWs (8-10). Usually, Raman spectra are acquired with an optical microscope attached to the Raman spectrometer, under such conditions the excitation is done with a relative high power laser density, the energy absorbed by the NWs cannot be efficiently dissipated and the NWs can reach elevated temperatures that broaden and downshift the one phonon Raman bands, inducing misinterpretation of the Raman spectrum if one only considers the phonon confinement. Therefore, the thermal effects have to be considered for a straightforward interpretation of the Raman data, beyond the mere phonon confinement effect. 
For this purpose a careful analysis of the temperature induced by the laser beam on excited NWs is required.

We present herein a study of the Raman spectra of Si and alloyed $\mathrm{Si}_{\mathrm{x}} \mathrm{Ge}_{1-\mathrm{x}} \mathrm{NWs}$, revealing the influence of the laser induced heating on the Raman spectrum. The temperature distribution inside the NWs is modelled using finite element analysis for solving the heat transport equation. The spatially resolved Raman spectrum of NWs was measured, and the data are interpreted in terms of the heating induced by the laser beam.

\section{EXPERIMENTAL AND SAMPLES}

NWs ( $\mathrm{Si}$ and $\mathrm{Si}_{\mathrm{x}} \mathrm{Ge}_{1-\mathrm{x}}$ ) were grown by the vapour liquid solid (VLS) method in a low pressure chemical vapour deposition (LPCVD) reactor on (100) Si substrates using $\mathrm{Si}_{2} \mathrm{H}_{6}$ and $\mathrm{GeH}_{4}$ as the precursor gases. The Si substrates were dipped in HF, rinsed for $30 \mathrm{~s}$ in APTES, dried with $\mathrm{N}_{2}$ and subsequently rinsed in a colloidal suspension of $\mathrm{Au}$ particles $(30 \mathrm{~nm}$ in diameter) for $180 \mathrm{~s}$ for the $\mathrm{Si} \mathrm{NWs}$. The growth was carried out at $500^{\circ} \mathrm{C}$ using $\mathrm{Si}_{2} \mathrm{H}_{6}$ as a precursor gas with a $10 \mathrm{sccm}$ flow; $\mathrm{N}_{2}$ was used as a carrier gas with a flow of $90 \mathrm{sccm}$, and a total pressure of $400 \mathrm{mTorr}$. For the SiGe NWs, a $3 \mathrm{~nm}$ thick Au layer was deposited, then the samples were loaded into the LPCVD reactor, and annealed at $500{ }^{\circ} \mathrm{C}$ for 1 hour in $\mathrm{H}_{2}$ atmosphere ( $400 \mathrm{mTorr}, 100 \mathrm{sccm}$ ) forming the Au-Si droplets. The NWs were grown at $500{ }^{\circ} \mathrm{C}$ during 30 min, adjusting the $\mathrm{GeH}_{4}$ and $\mathrm{Si}_{2} \mathrm{H}_{6}$ flows in such a way that the flow ratio $\mathrm{GeH}_{4}: \mathrm{Si}_{2} \mathrm{H}_{6}$ was varied between 0.25 and 10 for the growth of alloyed NWs, and a flow of $\mathrm{Si}_{2} \mathrm{H}_{6}$ of $10 \mathrm{sccm}$ for the $\mathrm{Si} \mathrm{NWs;} \mathrm{N}_{2}$ was the carrier gas with a flow of $90 \mathrm{sccm}$; the total pressure was maintained at 400 mTorr.

The Raman spectra were acquired with a Labram UV-HR 800 Raman spectrometer from Jobin Yvon. The excitation was done with either an $\mathrm{Ar}^{+}$laser $(514.5 \mathrm{~nm})$ for $\mathrm{Si} \mathrm{NWs}$ or a He-Ne laser $(632.8 \mathrm{~nm})$ for SiGe NWs, the laser powers on the focal plane were in the range of $50 \mu \mathrm{W}$, focused through a microscope objective, giving a Gaussian power distribution. The spectra were acquired on both bundles of NWs anchored to the substrate, and individual NWs detached from the substrate and deposited on a carbon lacey grid holder as those used for electron microscopy.

\section{RESULTS AND DISCUSSION}

In previous reports was demonstrated that heating induced by the Raman beam has a relevant contribution to the Raman spectrum of NWs (8-10). The Raman spectrum can be acquired over a bundle of NWs, or can be acquired on individual NWs conveniently separated from the substrate, deposited and localized in the grid holder in the scanning electron microscope (SEM). One of the main problems regarding the acquisition of the Raman spectrum of the NWs concerns the fact that the NWs cannot be observed in the optical microscope, upmost, one can observe diffuse light dispersion images on bundles of NWs, but the exact position of the NWs inside the laser beam cannot be determined, because the size of the NWs is below the resolution of the optical microscope. The Raman scattering volume is partially filled by NWs, with different diameters and lengths, constituting a very inhomogeneous scattering volume. See the SEM image of the NWs in Fig. 1, the circle represents the laser beam. In the SEM image one observes diameter dispersion, and NWs several micrometers long. The diameter of the NWs is larger than the diameter for which the phonon confinement becomes relevant to shape the one 


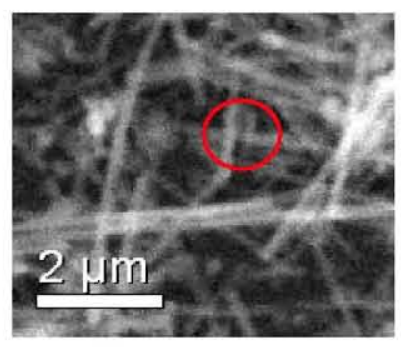

Fig.1. SEM image of the NWs, the red circle represents the laser beam

phonon Raman band (4). In the case of Si NWs, where no composition effects should be considered, the Raman spectrum of bundles of NWs, Fig. 2, is anomalously broad, which can only be interpreted according to the laser induced heating; in fact, the diameter dispersion can contribute to the phonon lineshape in two ways: the phonon confinement, only when the diameters are smaller than $20 \mathrm{~nm}$, or because of the different temperature reached depending on their dimensions and the amount of excitation energy absorbed. Note that the laser spot at focus has a diameter $\sim 1 \mu \mathrm{m}$, observing the SEM image of the NWs, Fig.1, one can deduce that the laser beam is probing a reduced number of wires.

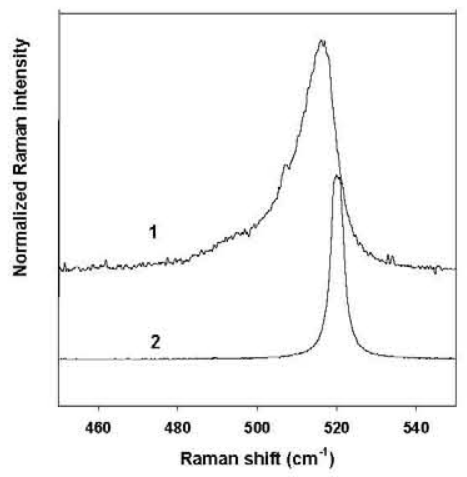

Fig.2. Raman spectrum of a bundle of NWs (1), and of a control bulk Si substrate (2)

The phonon confinement model does not account for the observed phonon bands, which are anomalously large, and cannot be fitted by the Campbell-Fauchet model (4), which is consistent with the large diameter observed in the SEM images. For the NWs with diameters larger than the one required to observing the phonon confinement effects, the main factor contributing to the shift and broadening of the phonon band is the heating of the NWs under the excitation with the laser beam (8-10). The Raman band shifts to lower wavenumbers and broadens with the increase of temperature, becoming more symmetric with respect to the Raman lineshape due to pure phonon confinement; the effect of temperature and diameter on the peak frequency and full width at half maximum, are shown in Fig. 3, as calculated from the temperature dependence of the Si phonon band reported in ref.11, and using the Campbell and Fauchet model (4) respectively. The experimental full width at half maximum (FWHM) of the bundle of NWs is anomalously large, it does not agree with the results expected for a single NW at high temperature. The discrepancies between the experimental and the calculated Raman parameters arise from the non homogeneous temperature inside the scattering volume, which, a priori, should be due to the dispersion in the dimensions of the NWs probed by the laser beam. 

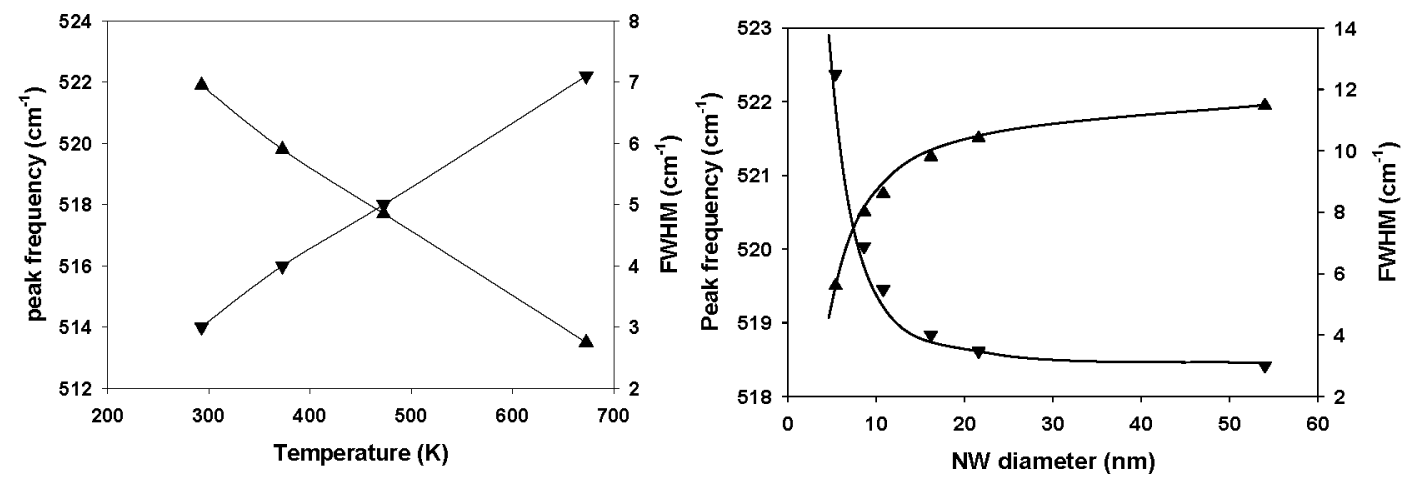

Fig.3. Peak frequency ( $\boldsymbol{\Delta}$ ), and FWHM ( $\boldsymbol{\nabla}$ ) vs T for bulk Si (left), and NW diameter (right) respectively. The experimental parameters corresponding to the spectrum of the bundle of NWs of Fig. 2 are $513 \mathrm{~cm}^{-1}$ (peak frequency), and $15 \mathrm{~cm}^{-1}$ (FWHM) respectively. The diameters of the NWs measured in this work are $\cong 100 \mathrm{~nm}$.

In order to study the temperature distribution induced by the laser beam inside the NWs, we carried out finite element analysis of the heat dissipation in the NWs under the exposure to the focused laser beam with a Gaussian power profile. The calculation took account of the dependence of the thermal conductivity with the NWs diameter, the dimensions of the NW and its position inside the laser beam spot. Two factors have to be considered, the thermal transport and dissipation, and the laser energy absorbed by the NWs. Concerning the absorbed energy, one considers the laser power and wavelength; the heat dissipation is determined by the thermal conductivity, which depends on the diameter of the NWs, and the surrounding media. For these calculations we considered a laser spot striking in the centre of the NWs, with a power of $50 \mu \mathrm{W}$. The NWs are surrounded by air, which has very poor thermal conductivity $(0.024 \mathrm{~W} / \mathrm{mK})$.

The temperature inside the region of the NW exposed to the laser beam depends on the NW diameter; therefore, if several NWs with different diameters are simultaneously excited by the laser beam significant temperature differences can be reached in between them; in this context one can consider the scattering volume as very inhomogeneous from the thermal point of view. On the other hand, the axial temperature variation inside the laser beam in a single NW does not exceed $10^{\circ} \mathrm{C}$, which rules out the existence of very large temperature gradients inside a NW. For NWs anchored to the substrate one observes a marked thermal asymmetry; however, in the zone excited by the laser beam the temperature variation is only a few ${ }^{\circ} \mathrm{C}$, which gives temperature gradients below $10^{\circ} \mathrm{C} / \mu \mathrm{m}$, which cannot account by itself for the anomalous broadening of the phonon bands. These considerations provide a route for the interpretation of the Raman spectra of discrete sets of Si NWs with dispersed diameters, which reach different temperatures under the exposure to the laser beam. The spectrum of Fig. 2 was fitted by the convolution of the Raman spectra of $4 \mathrm{NWs}$ with diameters of $20,30,40$ and $100 \mu \mathrm{m}$, which according to our calculations reach temperatures of $400,325,250$ and $150^{\circ} \mathrm{C}$, respectively, obtaining a reasonable fitting of the experimental data [10]. Nevertheless, the non possibility of observing the true disposition of the NWs inside the laser beam makes very difficult to achieve a straightforward interpretation of the data. The only way to achieve an unambiguous interpretation of the Raman spectrum consists of the study of individual NWs.

Fig. 4 shows the results of the simulation of the maximum temperature reached by a $\mathrm{Si}$ $\mathrm{NW}, 6 \mu \mathrm{m}$ long and $115 \mathrm{~nm}$ diameter, when the NW is transversally crossed by the laser spot. In this case, following the experimental set-up, the NW has been considered on top of a grid holder, 
where only a line of the cylinder of the NW is in contact with the holder; thus, we assume that the heat dissipation through it is nearly negligible. In order to elucidate the effect of the position of the laser beam with respect to the ends of the $\mathrm{NW}$, the incidence point has been situated at two positions, corresponding to the middle of the NW (A) and close to one of the ends (B) $(1 \mu \mathrm{m}$ away). There are almost no differences between the two laser beam positions along the NW, which is due to the short length of the NW in this case, and the fact that it was free standing. The differences are more marked in the case of longer NWs, and NWs anchored to the substrate. What is relevant is the great influence of the position of the NW inside the laser spot on the temperature reached by the NW; when the NW is in the centre of the spot exceeds by $440^{\circ} \mathrm{C}$ the temperature with respect to the NW just tangent to the laser beam. This is a very important point when one measures bundles of NWs, because in that case, the temperatures reached by the different NWs not only depends on the dimensions of the NWs, but also on where is each of them located with respect to the laser beam. The experimental results for SiGe NWs fully corroborate this assertion.

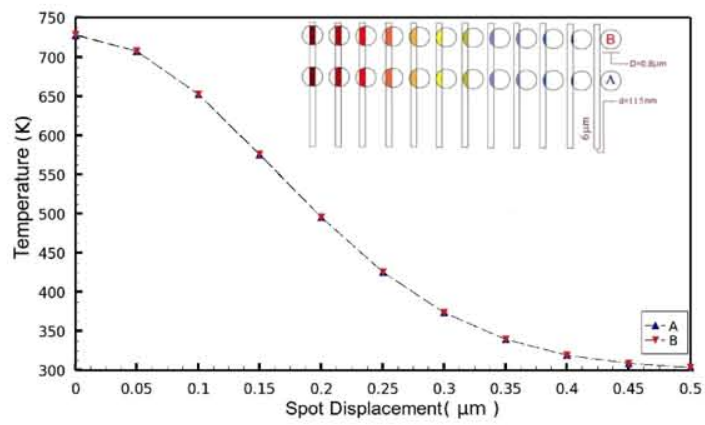

Fig.4. NW temperature as a function of the laser spot position for a Si NW.

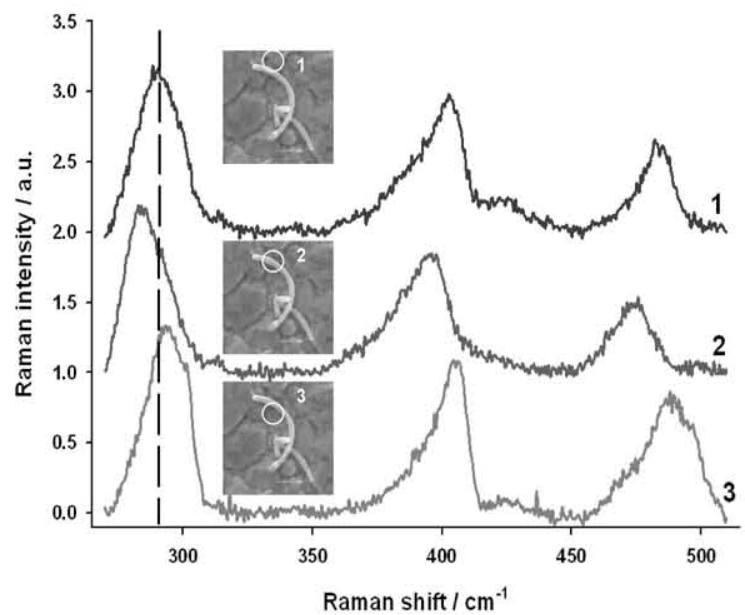

Fig.5. Raman spectra of the SiGe NW shown in the micrograph acquired at three points separated each other $0.25 \mu \mathrm{m}$. The white circles represent the positions of the laser beam. The vertical line is a guide to the eye to follow the shift to the low frequency when the NW is in the center of the laser beam (2), it peaks at higher frequency when the NW is excentric with the laser beam, (1) and (3).

The SEM image of a free standing $\mathrm{Si}_{\mathrm{x}} \mathrm{Ge}_{1-\mathrm{x}} \mathrm{NW}$ deposited on the grid holder is shown in Fig.5. This NW was scanned by the laser beam and the spectrum acquired at different positions. The alloyed NWs present an additional problem concerning the composition, which may be not 
homogeneous along the wire. In our alloyed NWs, Ge segregation is observed, which can contribute to make compositionally inhomogeneous the NWs (12). Raman maps were acquired, with a step size of $0.25 \mu \mathrm{m}$. The spectra are characterized by a broad peak consisting of two components, corresponding to the Ge-Ge vibrations of both the alloy and the segregated Ge, the broad band related to Si-Ge vibrations, also evidencing two contributions, and a band corresponding to Si-Si vibrations, which only presents two components in selected zones. The splitting of the bands may be due to the inhomogeneous composition of the NWs, because of the oxidation (12). The Ge composition was deduced from the intensity ratio $\mathrm{I}_{\mathrm{Ge}-\mathrm{Ge}} / \mathrm{I}_{\mathrm{Si}-\mathrm{Si}}(13)$. We have tested this relation with a batch of epitaxial SiGe layers of known compositions, obtaining very good agreement. The peak positions, also related to the Ge composition (13) (assuming that the NWs are relaxed), was not used for this purpose, since they are expected to be affected by the laser heating. The Raman spectra obtained on three adjacent steps evidence relative peak shifts, Fig.5, which at such a short scale can only be accounted by the dependence of the temperature reached by the NW with the relative position of the NW with respect to the laser beam; in fact the composition was estimated as constant, $\mathrm{x} \cong 0.53$, at the scale of variation of the positions where the three spectra were acquired, see the micrograph of Fig.5.

\section{CONCLUSION}

$\mathrm{Si}$ and $\mathrm{Si}_{1-\mathrm{x}} \mathrm{Ge}_{\mathrm{x}} \mathrm{NWs}$ have been characterized by micro-Raman spectroscopy. The results obtained evidence that heating, induced by the excitation laser beam, has a relevant contribution

to the phonon lineshape. Solving the heat transport equation by finite element analysis permits to have a picture of the temperature distribution in the NWs depending on the excitation conditions. According to these results he Raman spectrum of a bundle of NWs presents a high degree of uncertainty.

Acknowledgements This work was funded by the Spanish Government (Grant No. MAT-2007-66181, and MAT-2010-20441-C02) and by Junta de Castilla y León (Grant No. VA051A06 -GR202).

\section{REFERENCES}

[1] Y. Cui and C. Lieber, Science 291, 851 (2001).

[2] D.Li, Y.Wu, P.Kim, L.Shi, P.Yang, A.Majumdar; Appl.Phys.Lett. 832934 (2003)

[3] H. Richter, Z.P.Wang, L.Ley; Sol.St. Commun. 39, 625 (1981)

[4] I.H.Campbell, P.M. Fauchet; Sol. St. Commun. 58, 739 (1986)

[5] J. Jimenez, I. de Wolf, and J.P. Landesman, in "Microprobe Characterization of

Semiconductors"; vol.17 of Optoelectronic Properties of Semiconductors and Superlattices, ed. by J. Jimenez (Taylor and Francis, New York 2002), ch.2.R.

[6] S.Bhattacharyya, S.Samui ; Appl. Phys. Lett. 84, 1564 (2004)

[7] R.Gupta, Q.Xiong, C.K.Adu, U.J.Kim, P.C.Eklund; Nano Lett.3, 627 (2003)

[8] H.Scheel, S. Reich, A.C. Ferrari, M.Cantoro, A.Colli, C.Thomsen ; Appl. Phys. Lett. 88, 233114 (2006)

[9] K.W.Adu, H.R.Gutiérrez, U.J.Kim, P.C.Eklund; Phys.Rev.B 73, 155333 (2006)

[10] A. Torres, A. Martín-Martín, O. Martínez, A.C. Prieto, V. Hortelano, J. Jiménez, A.

Rodríguez, J. Sangrador, T. Rodríguez; Appl. Phys. Lett 96, 011904 (2010)

[11] H.H.Burke, I.P.Herman; Phys.Rev.B 48, 15016 (1993)

[12] C.Nishimura, G.Imammura, M.Fujii, T.Kawashima, T.Saitoh, S.Hayashi; Appl. Phys. Lett.93, 203101 (2008)

[13] J. C. Tsang, P. M. Mooney, F. Dacol, J. O. Chu. J. Appl. Phys. 75, 8098 (1994). 